\title{
Multimode quasi-phase-matching of high-order harmonic generation in gas-filled photonic crystal fibers
}

\author{
Patrick N. Anderson ${ }^{1}$, Florian Wiegandt ${ }^{1}$, Fei Y $u^{2}$, Daniel J. Treacher ${ }^{1}$, David T. Lloyd ${ }^{1}$, \\ Peter J. Mosley', Simon M. Hooker, Ian A. Walmsley ${ }^{I}$ \\ ${ }^{1}$ Clarendon Laboratory, University of Oxford, Parks Road, Oxford, OX1 3PU, UK \\ ${ }^{2}$ Centre for Photonics and Photonic Materials, Department of Physics, University of Bath, Bath, BA2 7AY, UK
}

Driving bright high-order harmonic generation (HHG) with few- $\mu \mathrm{J}$ pulses is a crucial step towards compact, high average power sources of coherent extreme ultraviolet (XUV) radiation for time-integrated applications including imaging. Unfortunately, reaching a sufficiently strong E-field to perform HHG with these pulses requires tight focusing, greatly reducing the interaction volume. An elegant solution to this problem is to restrict HHG to a hollow waveguide [1] and in particular a photonic crystal fiber [2]. Strong reabsorption in the XUV prohibits the use of multi-atmosphere pressures to achieve phase-matching [3], and instead quasi-phase-matching (QPM) is preferred. Here we demonstrate QPM of HHG for the first time within a gas-filled PCF.

We have developed several variants of the multimode-QPM (MM-QPM) scheme [4,5] applicable to PCFs. All of these utilize a "control" pulse coupled into a high-order mode that modulates either the amplitude or polarization of a "drive" pulse propagating in the fundamental. The dispersion characteristics of the fiber then dictate the modulation period and maximum interaction length. This was investigated experimentally by launching $800 \mathrm{~nm}, 30 \mathrm{fs}, 5 \mu \mathrm{J}$ "drive" and "control" pulses derived in a Mach-Zehnder interferometer into a 22.5 mm long section of hollow core negative curvature PCF [6]. The PCF spans two vacuum chambers: the first chamber is filled with 800 mbar of argon and the second chamber contains an XUV spectrometer maintained at high vacuum. Fig. 1(a) shows the spatially integrated intensity of $\mathrm{H} 19$ (30 eV) as a function of drive pulse delay. The large periodic enhancement in signal away from $t=0$ is consistent with our MM-QPM scheme, and the positioning of the peaks compares favorably with simulations. The enhancement in flux attributed to MM-QPM can be quantified (blue circle, Fig. 1(b-c)) by a factor of 60 at $30 \mathrm{eV}$.
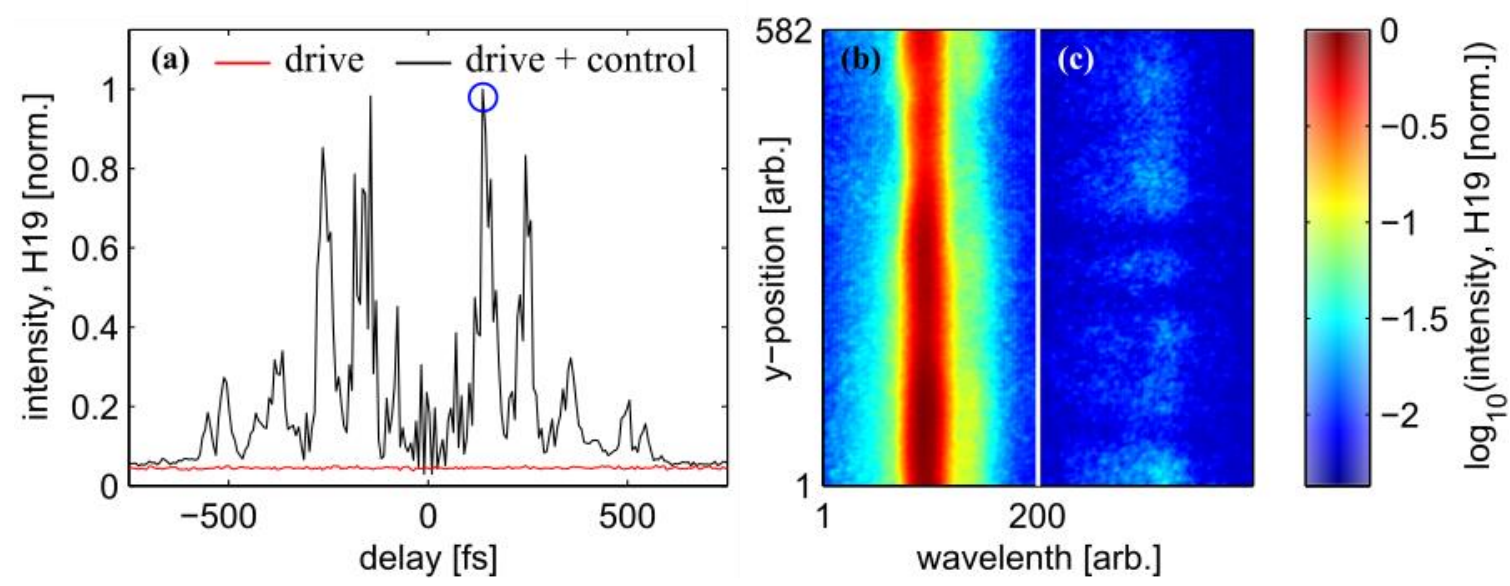

Fig. 1. (a) The intensity of the $19^{\text {th }}$ harmonic $(30 \mathrm{eV})$ recorded as a function of drive pulse delay. When the control pulse is introduced (black) significant enhancements in flux are observed along with a periodic structure consistent with MM-QPM. The spatio-spectral distribution at the highlighted delay (blue circle) is plotted with (b) and without (c) the control pulse. From this the enhancement in flux attributed to MM-QPM is estimated to be a factor of 60 at $30 \mathrm{eV}$.

\section{References}

[1] A. Rundquist, C. G. Durfee, Z. Chang, C. Herne, S. Backus, M. M. Murnane, and H. C. Kapteyn, "Phase-matched generation of coherent soft X-rays" Science 280, 1412-1415 (1998).

[2] O. H. Heckl, C. R. E. Baer, C. Kränkel, S. V. Marchese, F. Schapper, M. Holler, T. Südmeyer, J. S. Robinson, J. W. G. Tisch, F. Couny, P. Light, F. Benabid, and U. Keller, "High harmonic generation in a gas-filled hollow-core photonic crystal fiber" Appl. Phys. B 97, 369-373 (2009).

[3] T. Popmintchev, M. Chen, D. Popmintchev, P. Arpin, S. Brown, S. Ališauskas, G. Andriukaitis, T. Balčiunas, O. Mücke, A. Pugzlys, A. Baltuška, B. Shim, S. E. Schrauth, A. Gaeta, C. Hernández-García, L. Plaja, A. Becker, A. Jaron-Becker, M. M. Murnane, and H. C. Kapteyn, "Bright Coherent Ultrahigh Harmonics in the keV X-ray Regime from Mid-Infrared Femtosecond Lasers," Science 336, $1287-1291$ (2012).

[4] M. Zepf, B. Dromey, M. Landreman, P. Foster, and S. M. Hooker, "Bright Quasi-Phase-Matched Soft-X-Ray Harmonic Radiation from Argon Ions" Phys. Rev. Lett. 99, 143901 (2007).

[5] L. Z. Liu, K. O'Keeffe, and S. M. Hooker, "Quasi-phase-matching of high-order-harmonic generation using polarization beating in optical waveguides" Phys. Rev. A 87, 023810 (2013).

[6] F. Yu and J. C. Knight, "Spectral attenuation limits of silica hollow core negative curvature fiber" Opt. Express 21, 21466-21471 (2013). 